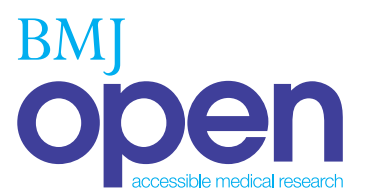

\title{
Transdiagnostic, affect-focused, psychodynamic, guided self-help for depression and anxiety through the internet: study protocol for a randomised controlled trial
}

To cite: Johansson $\mathrm{R}$, Hesser $\mathrm{H}$, Ljótsson B, et al. Transdiagnostic, affectfocused, psychodynamic, guided self-help for depression and anxiety through the internet: study protocol for a randomised controlled trial. BMJ Open 2012;2:e002167. doi:10.1136/bmjopen-2012002167

- Prepublication history for this paper are available online. To view these files please visit the journal online (http://dx.doi.org/10.1136/ bmjopen-2012-002167)

Received 27 September 2012 Revised 28 November 2012 Accepted 29 November 2012

This final article is available for use under the terms of the Creative Commons Attribution Non-Commercial 2.0 Licence; see http://bmjopen.bmj.com

For numbered affiliations see end of article.

Correspondence to Robert Johansson; robert.johansson@liu.se

\section{ABSTRACT}

Introduction: Cognitive behaviour therapy delivered in the format of guided self-help via the internet has been found to be effective for a range of conditions, including depression and anxiety disorders. Recent results indicate that guided self-help via the internet is a promising treatment format also for psychodynamic therapy. However, to date and to our knowledge, no study has evaluated internet-delivered psychodynamic therapy as a transdiagnostic treatment. The affect-phobia model of psychopathology by McCullough et al provides a psychodynamic conceptualisation of a range of psychiatric disorders. The aim of this study will be to test the effects of a transdiagnostic guided self-help treatment based on the affect-phobia model in a sample of clients with depression and anxiety.

Methods and analysis: This study will be a randomised controlled trial with a total sample size of 100 participants. The treatment group receives a 10-week, psychodynamic, guided self-help treatment based on the transdiagnostic affectphobia model of psychopathology. The treatment consists of eight text-based treatment modules and includes therapist contact in a secure online environment. Participants in the control group receive similar online therapist support without any treatment modules. Outcome measures are the 9-item Patient Health Questionnaire Depression Scale and the 7-item Generalised Anxiety Disorder Scale (GAD-7). Process measures that concerns emotional processing and mindfulness are included. All outcome and process measures will be administered weekly via the internet and at 6-month follow-up.

Discussion: This trial will add to the body of knowledge on internet-delivered psychological treatments in general and to psychodynamic treatments in particular. We also hope to provide new insights in the effectiveness and working mechanisms of psychodynamic therapy based on the affect-phobia model.

\section{ARTICLE SUMMARY}

Article focus

- Depression and anxiety disorders are major health problems, which lower the quality of life for the individual and generate large costs for society.

- Internet-delivered cognitive behaviour therapy in the form of guided self-help are effective in the treatment of depression and anxiety disorders.

- Psychodynamic therapy via the internet seem effective for specific disorders, but has not been evaluated as a transdiagnostic internet-delivered treatment.

- A transdiagnostic, affect-focused, psychodynamic, internet-delivered guided self-help will be tested in a sample of participants with depression and anxiety disorders.

Key messages

- Treatments based on the affect-phobia model of psychopathology have not previously been evaluated as guided self-help delivered via the internet.

- Potential processes of change will be measured during treatment and mechanisms of the treatment will be investigated. This will contribute both to the field of psychodynamic treatments and to that of internet-delivered psychological treatments in general.

Strengths and limitations of this study

- This randomised controlled trial will compare the effectiveness of an affect-focused, psychodynamic intervention to an active control condition.

- A sample of 100 participants will be used to ensure sufficient power.

- Mechanisms of change will be investigated using state-of-the-art methods for evaluating longitudinal mediation.

\section{INTRODUCTION}

Depression and anxiety disorders are major health problems, which lower the quality of 
life for the individual and generate large costs for society. ${ }^{1}{ }^{2}$ Lifetime prevalence for mood disorders and anxiety disorders have been estimated to be $20.8 \%$ and $28.8 \%$, respectively. ${ }^{3}$ Treatment alternatives include various psychotherapies, among which cognitive behaviour therapy (CBT) has an established strong empirical base. ${ }^{45}$ However, psychodynamic therapy has also shown promising results for the treatment of depression, ${ }^{6}$ panic disorder $^{7}$ and generalised anxiety disorder (GAD) ${ }^{8}$ During the last decade, there has been a significant progress in research concerning psychological guided self-help treatments and internet-delivered psychotherapies. ${ }^{9} 10$ Recent meta-analyses have shown that Internet-delivered CBT (ICBT), given as guided selfhelp, and face-to-face psychotherapy, is equally effective in the treatment of depression and anxiety disorders. ${ }^{11}$

Almost all published studies in the field of internetdelivered psychological treatments have endorsed a CBT approach. However, results from two recent randomised trials concerning depression and GAD indicate that psychodynamic therapy can also be delivered via the internet. $^{12} 13$

\section{Transdiagnostic and unified treatments}

A large portion of evidence-based psychological treatments target specific conditions, for example, depression and social anxiety disorder. There are also transdiagnostic and unified protocols where the treatment material has been arranged to fit a broader range of patients. ${ }^{14}$ Typically, the interventions in these protocols are based on psychological treatment components that target common behavioural patterns in various disorders, such as avoidance behaviours and maladaptive thought patterns, using for example, exposure, cognitive restructuring and emotion regulation techniques. ${ }^{14}$ Transdiagnostic ICBT protocols have been developed and tested with promising results among clients with anxiety disorders and depression. ${ }^{15} 16$

\section{Affect-phobia treatment and affect-focused psychodynamic treatment}

McCullough et $a l^{17}$ described a treatment model which is an example of a unified treatment grounded in psychodynamic theory. The model, called affect-phobia treatment (APT), follows the fundamental structure of psychodynamic psychotherapy as outlined by Malan's triangle of conflict (ie, defences and anxieties block the expression of feelings) and triangle of person (ie, work with conflicts in relation to the therapist and current and past persons). ${ }^{18}$ In a psychodynamic therapy based on the APT model, the therapist typically clarifies defences, helps the client to observe and experience the underlying affects and helps the client to regulate associated anxiety. ${ }^{17}$ Formally, the treatment includes three main treatment objectives: defence restructuring (recognising and relinquishing maladaptive defences), affect restructuring (desensitisation of affects through exposure to conflicted feeling) and self/other restructuring (improvement in sense of self and relationship with others). In summary, the main goal of psychodynamic psychotherapy based on the APT model is to help clients experience and to adaptively express feelings, that have been previously avoided. ${ }^{17}$

Two randomised trials have investigated the efficacy of APT for personality disorders. ${ }^{19} 20$ These trials showed that APT can be effective in reducing general psychiatric symptoms. However, except for case-series and some small uncontrolled studies, ${ }^{21}$ to date no trial has investigated the efficacy of APT for patients with a primary Axis I disorder.

\section{Processes of change}

To develop more effective psychotherapeutic treatments, it is crucial to understand the mechanisms of change that contribute to improvement in therapy. However, the mechanisms that cause therapeutic change are still largely unknown. In the experiential and psychodynamic traditions, including APT, experiencing and processing of the affective experience is considered central to therapeutic change. ${ }^{22} 23$ Typically, the therapist in APT helps the client recognise maladaptive defences, regulate anxiety and gradually experience feelings through systematic desensitisation to the conflicted emotions. ${ }^{17}$ Thus, emotional processing seems to be a relevant concept for understanding psychological treatments based on the APT model.

\section{Main research questions and hypotheses}

1. Is a transdiagnostic, affect-focused, psychodynamic, internet-delivered, guided self-help approach effective in reducing symptoms of depression and anxiety, when compared with an active control condition?

2. Do the degrees of emotional processing and mindfulness change more in the treatment condition than in the active control condition?

3. Do the degrees of emotional processing and mindfulness mediate symptom change on depression and anxiety measures?

\section{METHODS AND ANALYSIS}

\section{Design of the study and ethics statement}

The current study will be a randomised controlled trial with a sample size of 100 participants. The study protocol was approved by the regional Ethics Review Board in Linköping, Sweden. Written informed consent will be obtained from all participants using an online screening environment.

\section{Study population}

Patients will be recruited from the community via advertisements in newspapers and on the internet. To be included in the study, the participant must be at least 18 years of age and fulfil a principal DSM-IV diagnosis of either depression, social anxiety disorder, panic disorder or GAD. The Mini-International Neuropsychiatric Interview $\left(\mathrm{MINI}^{24}\right)$ will be used to diagnose DSM-IV 
disorders. Last-semester MSc students from a 5-year clinical psychology programme, who have been trained in the diagnostic procedure, will conduct the interviews by telephone. In addition, the participant must score at least 10 on either the 9-item Patient Health Questionnaire Depression Scale $\left(\mathrm{PHQ}-9^{25}\right)$ or the 7-item Generalised Anxiety Disorder Scale $\left(\mathrm{GAD}-7^{26}\right)$. Participants with a principal diagnosis of post-traumatic stress disorder or obsessive-compulsive disorder will be excluded. This restriction is due to the fact that the APT model does not conceptualise these disorders as phobias of affect, and hence, the treatment material used is not assumed to be applicable in these cases. ${ }^{17}$ Additional exclusion criteria include suffering from a severe psychiatric conditions such as bipolar disorder or psychosis; risk of suicide, assessed by the $\mathrm{MINI}^{24}$ alcohol or drug abuse and ongoing participation in any psychotherapy. Moreover, if the participant is receiving psychotropic medication, the dosage must have been stable for at least 3 months prior to the start of the treatment.

\section{Sample size}

A power calculation ${ }^{27}$ using a linear mixed-effects model (10 time points with an autoregressive error structure with a random intercept and slope) showed that approximately 46 (46.3) participants in each condition would be sufficient to detect a moderate between-group effect size of Cohen's $d=0.50$ ( $\alpha$ level $=0.05$ ) with $80 \%$ power. Assuming a total attrition rate of $10 \%$ (at equal rate across time and condition), a similar power calculation estimated sample size to be approximately 51 (51.3) participants per condition. Given these results, we aim to include 100 participants in the study.

\section{Interventions}

The treatment will be given as guided self-help with minimal text-based guidance provided on a weekly basis. This treatment format is an established way of delivering psychological treatments for depression and anxiety disorders. $^{911}$ The text material used in the treatment modules will be based on the book 'Living Like You Mean It' by Ronald J Frederick, ${ }^{28}$ which is theoretically grounded in psychodynamic theory and affective science and follows a similar structure as the original APT manual. There are eight modules in total, which all contain homework exercises that a client needs to complete before proceeding to the next module.

This treatment emphasises the practice of mindfulness more than in traditional psychodynamic therapy. Throughout treatment, the client is taught how to apply mindfulness in the process of identifying, attending to, and being present with emotional experience. In the context of the APT model, practicing 'emotional mindfulness' can be one way of learning how to be aware of, experience and tolerate emotions. The treatment teaches the client to gradually develop mindful presence as a response to the physical manifestation of emotions which, within the APT model, can be considered as exposure to one's feelings. All interventions are grounded in the affect-phobia model illustrated by the conflict triangle: maladaptive defences are identified and relinquished, anxiety is regulated, and warded off feelings are approached and experienced. After experiencing one's core feelings, the client is encouraged, when appropriate, to express these feelings in interpersonal contexts. Expressing feelings to others is seen as essential to shifting both the sense of self and others. Detailed steps of the treatment are described in figure 1 .

The online therapists will have contact with clients using a secure online environment resembling email, but with enhanced security. This mode of communication follows a standard procedure used in a range of studies investigating ICBT. $^{9}$ The primary role of the therapists will be to give feedback on the clients' exercises and experiences, and to administer gradual access to the treatment modules. In addition, the therapists will provide support, clarifications of the material and other forms of help that will enable the clients to complete

Module 1. Introduction and problem formulation using the affect-phobia model.

Module 2. Historical understanding and explanation of the problem described.

Module 3. Mindfulness practice to start approaching emotional experience.

Module 4. Defense restructuring.

Module 5. Anxiety regulation techniques.

Module 6. Affect experiencing techniques.

Module 7. Affect expression and self/other restructuring.

Module 8. A summary of the previous material and advice for continued work.

Figure 1 Description of the modules in the treatment. 
the material on time. The therapists will answer questions once every day, during weekdays. All therapists will be supervised biweekly by Ronald J. Frederick, the author of the book on which the treatment is based.

The therapists will be three last-semester MSc students from a 5-year clinical psychologist programme. All therapists will have a clinical training in affect-focused psychodynamic psychotherapy and treatment experience using this method.

Participants in the active control group will not receive any text-based treatment modules. They will, however, receive online therapist contact, where the therapists will provide support but will not use any specific psychological techniques other than basic therapeutic skills such as empathic listening and asking for clarifications. A similar active control condition has been used in previous treatment studies. ${ }^{12}$

\section{Randomisation and procedure}

After applying to the study, the participant will be directed to an online screening consisting of demographic questions and of online versions of the clinical outcome measures and the process measures (see below). These results will later be used as a pretreatment assessment. A participant will be contacted for a telephone-based diagnostic interview if he or she has completed the screening and meets the initial inclusion criteria (a score of at least 10 on either the PHQ-9 or the GAD-7). The interview consists of diagnostic interview questions about depression and anxiety disorders in addition to questions about use of medications and psychological treatments. The principal diagnosis assessed during the interview will be recorded. Psychiatric diagnoses are confirmed using the MINI. ${ }^{24}$ The participants will be allocated to one of the two groups in a 1:1 ratio using block randomisation. An independent person, separate from the staff conducting the study, will handle the randomisation using an online randomisation tool.

\section{Assessments}

Clinical outcome will be measured both by self-report questionnaires and a clinical interview. Mechanisms of change will be measured by two self-report measures. Clinical and process self-report measures will be administered weekly during the treatment phase. At the 6-month follow-up, a clinical interview will be conducted in addition to self-report measures.

\section{Clinical outcome measures}

Measures of depression and anxiety will be the PHQ- $-9^{25}$ and the GAD-7. ${ }^{26}$ The PHQ-9 is an established measure of depression which contains nine items, each scored $0-3$ and with a total score in the range of $0-27$. It has been shown to have good psychometric properties (Cronbach's $\alpha$ in the range 0.86-0.89). ${ }^{29}$ The GAD-7 was originally developed to diagnose GAD, but has also proved useful in detecting and assessing severity of panic, social anxiety and post-traumatic stress disorder. ${ }^{26}$
Psychometric properties have been proved to be good also for the GAD-7 (Cronbach's $\alpha=0.92$ ) ${ }^{29}$ The choice to administer outcome and process measures weekly, prevented the inclusion of further measures of anxiety.

The combination of PHQ-9 and GAD-7 for measuring depression and anxiety have been used in several treatment trials, for example, in the implementation of psychological therapies for anxiety and depression in British routine practice, which includes data from 7859 patients. ${ }^{30}$ Both the PHQ-9 and the GAD-7 are considered primary outcome measures in the study.

In addition to the self-report measures, clinical outcome will be measured in a clinical interview via telephone. Psychiatric diagnoses will be assessed at posttreatment and at follow-up using the MINI. The interview also aims to give an estimate of global improvement, measured by the seven-point version of the Clinical Global Impression-Improvement scale. ${ }^{31}$ Assessors of outcome in the clinical interview will be blind to treatment condition.

\section{Process measures}

In affect-phobia therapy, emotional processing is assumed to be related to symptom change. To explore this further in the current study, the Emotional Processing Scale (EPS-25) ${ }^{32}$ will be used to assess emotional processing deficits and the process of emotional change during treatment. The measure contains five subscales, three of these relate to emotional control or dysregulation (emotional avoidance, suppression, unregulated emotion, one subscale (impoverished emotional experience) shares some properties with the alexithymia construct ${ }^{32}$ and the last subscale (signs of unprocessed emotion) refers to persistent and intrusive emotional phenomena. The total score of the EPS-25 reflects the overall level of emotional processing deficit. $^{32}$ Psychometric properties for the EPS-25 has been found to be strong (Cronbach's $\alpha=0.92$ ) ${ }^{32}$ While the EPS-25 is primarily included to measure a specific change process, it is also considered a secondary outcome measure in this trial.

As described above, the treatment has a strong mindfulness component. Thus, the amount of mindfulness skills that the client acquires during treatment may be related to outcome. To assess the influence of mindfulness, the Five Facets of Mindfulness Questionnaire $\left(\mathrm{FFMQ}^{33}\right)$ is included to measure general mindfulness skills. The original FFMQ by Baer et a ${ }^{33}$ contains 39 items, while the Swedish version that will be used in this study consists of 29 items. ${ }^{34}$ The correlation between the Swedish FFMQ and the original FFMQ is $r=0.98 .{ }^{34}$ The FFMQ was constructed as a multifaceted assessment of mindfulness, measuring a general tendency to be mindful in daily life, which is defined by five factors: observing, describing, acting with awareness, nonjudging of inner experience and non-reactivity to inner experience. ${ }^{33}$ Psychometric properties have been proved to be good for the Swedish 29-item FFMQ (Cronbach's 
$\alpha=0.81) .{ }^{34}$ The FFMQ is considered a secondary outcome measure, besides being a process measure.

\section{Statistical analyses}

Differences in efficacy between the treatment and the active control group will primarily be investigated by modelling interaction effects of group and time. In order to adhere to the intention-to-treat principle, the outcome variables will be analysed using mixed effects models, given their ability to handle missing data. ${ }^{35}$ To examine processes of change in full detail using week-to-week measurements of both processes (ie, emotional processing and mindfulness) and outcomes, state-of-the-art methods for evaluating longitudinal mediation will be employed. ${ }^{36}$ Multiple analyses will be conducted in an exploratory fashion. This includes the testing of hypotheses that a change in a process variable at a specific time-point causes a change in an outcome variable at a later time-point. An exploratory approach will allow several hypotheses to be tested that will open up for further research in the field of mechanisms of change in affect-focused psychotherapy. All analyses will use full information maximum likelihood estimation, which provides unbiased estimates and correct standard errors in the presence of missing data, assuming data loss is missing for non-ignorable reasons. ${ }^{37}$

\section{DISCUSSION}

This study will test the efficacy of a transdiagnostic, psychodynamically oriented, 10-week, guided self-help treatment, based on an affect-phobia model of psychopathology. In addition, the study will explore working mechanisms of the treatment.

An important aim of the treatment tested is to stay close to the APT model. The conceptualisation of psychological problems are the same as in the original manual and the treatment is structured similarly. We assume that the essence of affect-phobia therapy is preserved in the guided self-help treatment. However, the treatments do differ in some aspects, for example, by not explicitly making use of transference relations. While we do not rule out that transference phenomena can occur, no interventions are included that address this. To our knowledge, no studies have investigated the efficacy of a similar treatment protocol via the internet. Hence, this study will contribute to the empirical base of internet-delivered psychological treatments in general and to that of psychodynamic approaches in particular. Moreover, no previous treatment study exists that evaluates the efficacy of face-to-face affect-phobia therapy for Axis I disorders using a randomised controlled trial. Therefore, this study also adds to the empirical base of psychodynamic therapies in general.

Investigating the mediating effects of emotional processing and mindfulness provides an opportunity to explore the mechanisms of change in affect-focused, psychodynamic therapy. The processes that contribute to therapeutic change are largely unknown. This study aims to explore how emotional processing is related to treatment outcome and thus tests the assumption that emotional processing is a crucial process in psychodynamic treatments based on the APT model. In addition, the role of mindfulness is similarly explored.

Moreover, there is a lack of psychodynamic therapy in other treatment formats than individual and group. Using the internet format of delivery makes it possible to reach individuals who lack access to therapists nearby, or who simply do not want to meet face-to-face. ${ }^{9}$

In addition, there are also benefits of using one transdiagnostic protocol compared with having several specific programmes. Clients with different disorders and comorbidities can be treated using the same protocol. This approach could potentially increase a scope of a treatment implementation in, for example, a psychiatric clinic or in primary care. By developing transdiagnostic protocols, the amount of therapist training could also potentially be reduced, as clinicians may need to learn fewer programmes.

\section{Author affiliations}

${ }^{1}$ Department of Behavioural Sciences and Learning, Linköping University, Linköping, Sweden

${ }^{2}$ Linnaeus Centre HEAD, Swedish Institute for Disability Research, Linköping University, Linköping, Sweden

${ }^{3}$ Division of Psychology, Department of Clinical Neuroscience, Division of Psychology, Karolinska Institutet, Stockholm, Sweden

${ }^{4}$ Center for Courageous Living, Beverly Hills, California, USA

${ }^{5}$ Psychiatry Section, Department of Clinical Neuroscience, Karolinska Institutet, Stockholm, Sweden

Contributors $\mathrm{RJ}$ in collaboration with $\mathrm{HH}$ and $\mathrm{GA}$ designed the study. $\mathrm{RJ}$ and $\mathrm{HH}$ drafted the manuscript. BL, RJF and GA reviewed and revised the manuscript. All authors have read and approved the final manuscript to be published.

Funding The study was supported in part by the Swedish council for working and life research and Linköping University (Professor contract).

Competing interests RJF is the author of the book used as a basis for the treatment manual.

Ethics approval The protocol was approved by the regional ethics board of Linköping, Sweden. Written informed consent will be obtained from all participants.

Provenance and peer review Not commissioned; externally peer reviewed.

\section{REFERENCES}

1. Ebmeier KP, Donaghey C, Steele JD. Recent developments and current controversies in depression. Lancet 2006;367:153-67.

2. Smit F, Cuijpers P, Oostenbrink J, et al. Costs of nine common mental disorders: implications for curative and preventive psychiatry. $J$ Ment Health Policy Econ 2006;9:193-200.

3. Kessler RC, Berglund P, Demler O, et al. Lifetime prevalence and age-of-onset distributions of DSM-IV disorders in the National Comorbidity Survey Replication. Arch Gen Psychiatry 2005;62:593-602.

4. Cuijpers P, van Straten A, Andersson G, et al. Psychotherapy for depression in adults: a meta-analysis of comparative outcome studies. J Consult Clin Psychol 2008;76:909-22.

5. Hofmann SG, Smits JAJ. Cognitive-behavioral therapy for adult anxiety disorders: a meta-analysis of randomized placebo-controlled trials. J Clin Psychiatry 2008;69:621-32.

6. Driessen E, Cuijpers P, de Maat SCM, et al. The efficacy of short-term psychodynamic psychotherapy for depression: a meta-analysis. Clin Psychol Rev 2010;30:25-36. 
7. Milrod $\mathrm{B}$, Leon $\mathrm{AC}$, Busch $\mathrm{F}$, et al. A randomized controlled clinical trial of psychoanalytic psychotherapy for panic disorder. $A m \mathrm{~J}$ Psychiatry 2007;164:265-72.

8. Leichsenring F, Salzer S, Jaeger U, et al. Short-term psychodynamic psychotherapy and cognitive-behavioral therapy in generalized anxiety disorder: a randomized, controlled trial. Am J Psychiatry 2009;166:875-81.

9. Andersson $\mathrm{G}$. Using the internet to provide cognitive behaviour therapy. Behav Res Ther 2009;47:175-80.

10. Johansson R, Andersson G. Internet-based psychological treatments for depression. Expert Rev Neurother 2012;12:861-70.

11. Cuijpers $P$, Donker T, van Straten A, et al. Is guided self-help as effective as face-to-face psychotherapy for depression and anxiety disorders? A systematic review and meta-analysis of comparative outcome studies. Psychol Med 2010;40:1943-57.

12. Johansson R, Ekbladh S, Hebert A, et al. Psychodynamic guided self-help for adult depression through the internet: a randomised controlled trial. PLoS One 2012;7:e38021.

13. Andersson G, Paxling B, Roch-Norlund $P$, et al. Internet-based psychodynamic vs cognitive behavioural guided self-help for generalized anxiety disorder: a randomised controlled trial. Psychother Psychosom 2012;81:344-55.

14. Barlow DH, Allen LB, Choate ML. Toward a unified treatment for emotional disorders. Behav Ther 2004;35:205-30.

15. Titov N, Andrews $\mathrm{G}$, Johnston L, et al. Transdiagnostic internet treatment for anxiety disorders: a randomized controlled trial. Behav Res Ther 2010;48:890-9.

16. Titov N, Dear BF, Schwencke G, et al. Transdiagnostic internet treatment for anxiety and depression: a randomised controlled trial. Behav Res Ther 2011;49:441-52.

17. McCullough L, Kuhn N, Andrews S, et al. Treating affect phobia: a manual for short-term dynamic psychotherapy. New York: Guilford Press, 2003.

18. Malan D. Individual psychotherapy and the science of psychodynamics. 2nd edn. Oxford: Butterworth-Heinemann, 1995

19. Winston A, Laikin M, Pollack J, et al. Short-term psychotherapy of personality disorders. Am J Psychiatry 1994;151:190-4.

20. Svartberg M, Stiles TC, Seltzer MH. Randomized, controlled trial of the effectiveness of short-term dynamic psychotherapy and cognitive therapy for cluster $\mathrm{C}$ personality disorders. Am J Psychiatry 2004:161:810-17.

21. Dornelas E, Ferrand J, Stepnowski R, et al. A pilot study of affect-focused psychotherapy for antepartum depression. J Psychother Integr 2010;20:364-82.
22. Greenberg LS, Pascual-Leone A. Emotion in psychotherapy: a practice-friendly research review. J Clin Psychol 2006;62:611-30.

23. Diener MJ, Hilsenroth MJ, Weinberger J. Therapist affect focus and patient outcomes in psychodynamic psychotherapy: a meta-analysis. Am J Psychiatry 2007;164:936-41.

24. Sheehan DV, Lecrubier $\mathrm{Y}$, Sheehan $\mathrm{KH}$, et al. The Mini-International Neuropsychiatric Interview (M.I.N.I.): the development and validation of a structured diagnostic psychiatric interview for DSM-IV and ICD-10. J Clin Psychiatry 1998:59:34-57.

25. Kroenke K, Spitzer RL, Williams JB. The PHQ-9: validity of a brief depression severity measure. J Gen Intern Med 2001;16:606-13.

26. Spitzer RL, Kroenke K, Williams JBW, et al. A brief measure for assessing generalized anxiety disorder: the GAD-7. Arch Intern Med 2006;166:1092-7.

27. Hedeker D, Gibbons RD, Waternaux C. Sample size estimation for longitudinal designs with attrition: Comparing time-related contrasts between two groups. J Educ Behav Stat 1999;24:70-93.

28. Frederick RJ. Living like you mean it: use the wisdom and power of your emotions to get the life you really want. San Francisco: Jossey-Bass, 2009.

29. Kroenke K, Spitzer RL, Williams JBW, et al. The patient health questionnaire somatic, anxiety, and depressive symptom scales: a systematic review. Gen Hosp Psychiatry 2010;32:345-59.

30. Richards DA, Borglin G. Implementation of psychological therapies for anxiety and depression in routine practice: two year prospective cohort study. J Affect Disord 2011;133:51-60.

31. Guy W. Clinical global impressions. ECDEU assessment manual for psychopharmacology. Rockville: NIMH, 1976.

32. Baker R, Thomas S, Thomas PW, et al. The Emotional Processing Scale: scale refinement and abridgement (EPS-25). J Psychosom Res 2010;68:83-8.

33. Baer RA, Smith GT, Hopkins J, et al. Using self-report assessment methods to explore facets of mindfulness. Assessment 2006;13:27-45

34. Lilja JL, Frodi-Lundgren A, Hanse JJ, et al. Five facets mindfulness questionnaire-reliability and factor structure: a Swedish version. Cogn Behav Ther 2011;40:291-303.

35. Gueorguieva R, Krystal JH. Move over ANOVA: progress in analyzing repeated-measures data and its reflection in papers published in the Archives of General Psychiatry. Arch Gen Psychiatry 2004;61:310-17.

36. MacKinnon DP. Introduction to statistical mediation analysis. New York: Lawrence Erlbaum Associates, 2008.

37. Little RJA, Rubin DB. Statistical analysis with missing data. 2nd edn New York: Wiley, 2002 